Relations industrielles

Industrial Relations

\title{
Insidious Workplace Behavior, Sous la direction de Jerald Greenberg, New York : Routledge, 2010, 374 p., ISBN : 978-1-84872-859-2.
}

\section{François Courcy}

Volume 66, numéro 4, automne 2011

URI : https://id.erudit.org/iderudit/1007645ar

DOI : https://doi.org/10.7202/1007645ar

Aller au sommaire du numéro

Éditeur(s)

Département des relations industrielles de l’Université Laval

ISSN

0034-379X (imprimé)

1703-8138 (numérique)

Découvrir la revue

Citer ce compte rendu

Courcy, F. (2011). Compte rendu de [Insidious Workplace Behavior, Sous la direction de Jerald Greenberg, New York : Routledge, 2010, 374 p., ISBN :

978-1-84872-859-2.] Relations industrielles / Industrial Relations, 66(4), 694-695.

https://doi.org/10.7202/1007645ar

Tous droits réservés (C Département des relations industrielles de l’Université Laval, 2011
Ce document est protégé par la loi sur le droit d'auteur. L'utilisation des services d'Érudit (y compris la reproduction) est assujettie à sa politique d'utilisation que vous pouvez consulter en ligne.

https://apropos.erudit.org/fr/usagers/politique-dutilisation/ 
relationships between national, sectoral and firm systems, as well as the coexistence of horizontal differentiation and unity across sectors and firms. The one area that the book does not cover is that of the individual employee; whether he or she is comfortable with these continued and renewed dualities, or whether underlying feelings of antipathy to employer demands are placing strains on the system from below. However, this gap is understandable within the context of this study, given that Keizer has his hands full simply to gather and interpret the data from his sample firms. But there is room here for further research, as the author no doubt already appreciates.

All in all, Keizer has done an excellent job in presenting such a balanced set of conclusions, skilfully avoiding the overblown rhetoric of previous studies that have hailed a complete transformation or collapse in lifetime employment in Japan. The book deserves to be read by scholars from the fields of management studies, economics, and sociology who are interested in contemporary employment practices in developed countries, and is an excellent example for graduate students who are themselves engaged in firm level field work. In short, this book is a welcome dose of good sense on a subject that routinely requires a grounding in real world data analysis in order to retain balance. I look forward to reading more from Keizer as he deepens and extends this research.

\section{Peter Matanle}

University of Sheffield

\section{Insidious Workplace Behavior}

Sous la direction de Jerald Greenberg, New York : Routledge, 2010, 374 p., ISBN : 978-1-84872-859-2.

Au cours des deux dernières décennies, les comportements antisociaux au travail ont reçu une attention sans cesse grandissante de la part des chercheurs et des professionnels de tous les horizons. Jerald Greenberg figure parmi les pionniers de ce domaine de recherche auquel il a contribué à maints égards en menant nombre de travaux précurseurs et en proposant des textes phares. En éditant Insidious Workplace Behavior, ce bâtisseur reconnu comme I'un des penseurs les plus influents en management donne la parole à une équipe de chercheurs chevronnés et émergents et de professionnels de premier plan. Les 10 chapitres formant I'ouvrage convergent tous pour souligner l'importance de porter sans délai une attention particulière aux conduites déviantes de nature insidieuse. Premier volume entièrement consacré au sujet, le propos n'en est pas moins bien documenté et étayé avec rigueur. Tour à tour, les chapitres décrivent en détail la nature et les formes les plus subtiles d'inconduites ainsi que les mécanismes de gestion efficace et les méthodes d'investigation scientifique.

Auteurs, chercheurs, professionnels et lecteurs informés ont tous été saisis au cours des dernières années par l'ampleur et la fréquence des agissements illicites survenant dans les systèmes sociaux et les organisations : homicides, scandales portant sur la corruption de dirigeants, fraudes financières et détournements d'actifs, etc. Ces actes ne passent d'ailleurs pas inaperçus et font régulièrement la une des journaux. Ils ne représenteraient toutefois que la pointe de l'iceberg des inconduites prévalant dans les milieux de travail. Pendant que ces actes manifestes captent l'attention, des conduites déviantes plus sournoises prévaudraient dans nos entreprises et institutions. Ces gestes omniprésents, mal intentionnés mais licites, systématisés mais en apparence anodins, au déploiement furtif mais à l'effet délétère sur les personnes et les systèmes, envahiraient le quotidien du travail. Ce sont les conduites insidieuses au travail. Dans un ouvrage incisif, 17 coauteurs lèvent sans détour le voile sur cette réalité malheureusement oblitérée par la présence récurrente d'inconduites plus manifestes. 
Au menu de l'ouvrage, le lecteur abordera dans un premier chapitre fort éclairant la nature exacte du concept de conduite insidieuse, construit défini en le distinguant des concepts usuels du domaine, comme l'agression, le vice ou la déviance. Ces clarifications permettent d'entreprendre dans une deuxième partie la lecture de six chapitres abordant autant de formes spécifiques de conduites insidieuses: I'agressivité, le sabotage, la vengeance, l'incivilité, l'humour sexiste et le mensonge. Chacun de ces chapitres comporte une section initiale définissant clairement le concept à l'étude et le distinguant de conduites analogues. Cette contribution n'est pas sans valeur, puisqu'il s'avère encore aujourd'hui difficile d'établir des distinctions précises entre certaines formes de déviance et la ligne entre l'acceptable et l'inacceptable au quotidien. Suivant ces clarifications, les principaux enjeux théoriques sont ensuite discutés en détail dans un langage sobre et accessible. Le tout demeure rigoureux et clairement articulé, rendant I'arrêt de la lecture souvent difficile. Enfin, l'étudiant et le chercheur y liront également de possibles programmations de recherche ou thèses stimulantes. Au terme de chacun des chapitres, on trouve enfin diverses méthodes d'intervention principalement destinées aux gestionnaires et aux victimes de ces actes néfastes.

Une troisième partie s'adresse principalement aux chercheurs ou aux professionnels de l'évaluation organisationnelle. Deux chapitres y traitent avec justesse des défis inhérents à l'investigation des conduites d'incivilité au travail et des enjeux propres aux méthodes de recherche dans ce domaine. Leurs propositions sont à la fois rigoureuses, sensées et créatives. Même si le gestionnaire se sentira moins interpelé par ces propos, il aurait intérêt à s'y immiscer. Car, si l'on a l'impression que ces conduites passent souvent inaperçues au quotidien, leur détection se complexifie rapidement lorsque l'on demande à son personnel ce qu'il a vu, subi ou commis. Les précieux conseils formulés dans le livre permettront une investigation à la fois éthique et systématique de plusieurs formes de conduites insidieuses.

Enfin, dans une dernière partie, l'éditeur conclut l'ouvrage par la proposition d'un calendrier de recherche fort bien documenté en étayant les questions, les défis et les enjeux auxquels chercheurs et théoriciens devront s'attaquer au cours des prochaines années. Chacune de ces propositions est discutée avec nombre d'appuis et d'explications. Sans l'ombre d'un doute, les défis ne manqueront pas!

En somme, ce premier volume sur le sujet éclaire tout un pan des inconduites plus subtiles survenant sur les lieux mêmes de notre travail. Ce faisant, I'ouvrage vise à stimuler, voire à accélérer, la réflexion et l'action dans un domaine déjà en effervescence. Cette contribution a le mérite d'offrir aux lecteurs de différents horizons et aux intérêts variés des méthodes, des outils et des réflexions pratiques ou théoriques fort utiles. Règle générale, le lecteur y trouvera son compte; certains pourraient toutefois rester quelque peu sur leur appétit. Quoique bien documenté, cet ouvrage n'aborde en effet qu'une poignée d'inconduites, délaissant nombre d'activités illicites tout aussi troublantes, comme celles liées au présentéisme ou au ralentissement de la production. II s'agit en quelque sorte d'une très belle invitation de laquelle devraient découler - si l'on se fie aux répercussions des travaux antérieurs de l'éditeur - un foisonnement d'activités et, progressivement, un élargissement des thématiques à inclure sous le parapluie des conduites insidieuses au travail. À la lecture de cet ouvrage collectif, les habitués de l'étude des conduites déviantes et antisociales au travail trouveront un nouveau souffle à leurs actions, réflexions ou investigations. Comme entrée en matière, ce livre offre une vision quelque peu déstabilisante d'une autre réalité du travail à laquelle il est difficile de rester insensible ensuite. En deux mots : une invitation irrésistible.

\section{François Courcy}

Université de Sherbrooke 